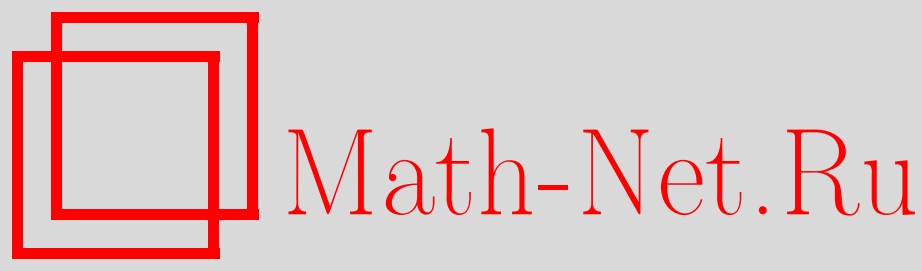

В. Д. Гордевский, Винтовые потоки с ускорением и уплотнением для модели твердых сфер, ТМФ, 2009, том 161, номер 2, 278-286

DOI: https://doi.org/10.4213/tmf6438

Использование Общероссийского математического портала Math-Net.Ru подразумевает, что вы прочитали и согласны с пользовательским соглашением http://www.mathnet.ru/rus/agreement

Параметры загрузки:

IP : 54.210 .77 .194

26 апреля 2023 г., 06:54:39

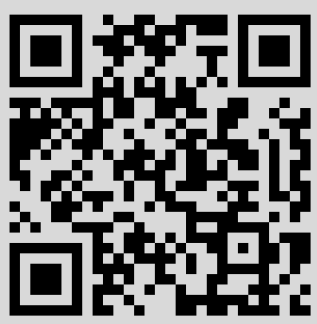




\section{ВИНТОВЫЕ ПОТОКИ С УСКОРЕНИЕМ И УПЛОТНЕНИЕМ ДЛЯ МОДЕЛИ ТВЕРДЫХ СФЕР}

Построена модель взаимодействия между двумя потоками в газе из твердых сфер, каждый из которых имеет структуру ускоряющегося и уплотняющегося винтового потока. Получены некоторые условия, достаточные для произвольной малости равномерно-интегральной, или "смешанной", нормы разности между левой и правой частями уравнения Больцмана.

Ключевые слова: твердая сфера, уравнение Больцмана, "смешанная" невязка, винтовой поток, ускорение-уплотнение.

\section{1. ВВЕДЕНИЕ}

В случае модели твердых сфер уравнение Больцмана имеет вид [1], [2]

$$
D(f)=Q(f, f),
$$

где

$$
\begin{gathered}
D(f)=\frac{\partial f}{\partial t}+v \frac{\partial f}{\partial x} \\
Q(f, f)=\frac{d^{2}}{2} \int_{\mathbb{R}^{3}} d v_{1} \int_{\Sigma} d \alpha\left|\left(v_{1}-v, \alpha\right)\right| \times \\
\times\left[f\left(t, v_{1}^{\prime}, x\right) f\left(t, v^{\prime}, x\right)-f\left(t, v_{1}, x\right) f(t, v, x)\right] .
\end{gathered}
$$

Здесь $f$ - искомая функция распределения молекул газа, $\partial f / \partial x$ (или $\left.f^{\prime}\right)$ - градиент по переменной $x ; t \in \mathbb{R}^{1}$ - время; $x=\left(x^{1} ; x^{2} ; x^{3}\right) \in \mathbb{R}^{3}$ и $v=\left(v^{1} ; v^{2} ; v^{3}\right) \in \mathbb{R}^{3}-$ координата и скорость молекулы; $d$ - ее диаметр; $\alpha \in \Sigma$, где $\Sigma \subset \mathbb{R}^{3}$ - сфера радиуса 1 с центром в нуле (здесь и далее $(a, b)=a b$ - скалярное произведение векторов $a, b \in \mathbb{R}^{3},(a, a)=a^{2}$ - скалярный квадрат вектора $\left.a \in \mathbb{R}^{3}\right)$. Связь между скоростями двух молекул до и после столкновения такова:

$$
v_{1}^{\prime}=v_{1}+\alpha\left(v-v_{1}, \alpha\right), \quad v^{\prime}=v-\alpha\left(v-v_{1}, \alpha\right)
$$

* Харьковский национальный университет им. В.Н. Каразина, Харьков, Украина. E-mail: gordevskyy2006@yandex.ru 
Для рассматриваемой модели взаимодействия между молекулами газа единственным точным решением уравнения (1), найденным в явном виде, до сих пор остается максвелловское распределение (максвеллиан)

$$
M=\rho\left(\frac{\beta}{\pi}\right)^{3 / 2} e^{-\beta(v-\tilde{v})^{2}} .
$$

Входящие в данное выражение гидродинамические параметры суть плотность газа $\rho$, его "обратная температура" $\beta=1 / 2 T$ ( $T$ - абсолютная температура; константа Больцмана для простоты полагается равной единице), $\tilde{v}$ - средняя, или массовая скорость молекул, которая может определенным образом зависеть от $t$ и $x$. Простейшие случаи, когда эти параметры постоянны (глобальный максвеллиан) либо зависят только от $x$ (стационарный неоднородный локальный максвеллиан, или винт в терминологии работы [3]) были известны еще Максвеллу и Больцману [1], [2], [4]-[6]. Наиболее общий (нестационарный) случай локальных максвеллианов был исследован в [2], [4], [5]; их описание можно найти также в [1], [6]. В статье [7] проведена детальная классификация таких решений и выяснены некоторые физические и геометрические особенности каждого из выделенных в этой работе типов локально-максвелловских распределений. Одно из описанных в работе [7] нестационарных, неоднородных точных решений уравнения Больцмана получило название ускоряющегося и уплотняющегося винта, ибо соответствующий ему поток газа не только вращается вокруг неподвижной оси с постоянной угловой скоростью, но также имеет растущие со временем плотность и линейную скорость вдоль указанной оси.

В работах [8]-[10] предложен подход, позволяющий искать явные приближенные решения уравнения Больцмана в виде бимодального распределения

$$
f=\sum_{i=1}^{2} \varphi_{i} M_{i}
$$

где глобальные максвелловские моды $M_{i}$ имеют вид $(5)$ с различными параметрами $\rho_{i}>0, \beta_{i}>0, \tilde{v}_{i} \in \mathbb{R}^{3}$, а коэффициентные функции $\varphi_{i}$ зависят от $t, x$ и предполагаются неотрицательными и гладкими, $i=1,2$. В качестве величины, характеризующей различие между левой и правой частями уравнения (1), используется равномерно-интегральная ("смешанная") невязка

$$
\Delta=\sup _{(t, x) \in \mathbb{R}^{4}} \int_{\mathbb{R}^{3}} d v|D(f)-Q(f, f)| .
$$

Аналогичная задача для случая стационарных винтовых потоков решена в работе [3], а для нестационарных вихрей (при этом $M_{i}, i=1,2$, представляют собой некие "квазимаксвеллианы" , являющиеся лишь приближенными решениями уравнения Больцмана) - в работе [11]. Использование в статьях [12], [13] иной, "чисто интегральной”, невязки $\Delta_{1}$ приводит к совершенно другому виду приближенных бимодальных решений, т.е. функций $\varphi_{i}, i=1,2$, в (6). В работе [14] в качестве 
максвелловских мод $M_{i}, i=1,2$, использованы новые точные нестационарные решения, которые соответствуют не вращающимся, но ускоряющимся и уплотняющимся потокам газа.

Целью настоящей работы является поиск таких распределений вида (6), где максвеллианы $M_{i}, i=1,2$, описывают винтовые потоки с ускорением и уплотнением, которые были бы приближенными решениями уравнения (1)-(4), т.е. позволяли бы сделать невязку (7) сколь угодно малой.

Постановка задачи такова. Пусть моды в (6) имеют вид [7]

$$
M_{i}=M_{i}(t, v, x)=\rho_{i}\left(\frac{\beta_{i}}{\pi}\right)^{3 / 2} e^{-\beta_{i}\left(v-\tilde{v}_{i}\right)^{2}},
$$

где

$$
\begin{aligned}
& \tilde{v}_{i}=\tilde{v}_{i}(t, x)=\bar{v}_{i}+\left[\bar{\omega}_{i} \times x\right]-\bar{u}_{i} t, \\
& \rho_{i}=\rho_{i}(t, x)=\bar{\rho}_{i} e^{\beta_{i}\left(\tilde{v}_{i}^{2}+2 \bar{u}_{i} x\right)}
\end{aligned}
$$

$\left([a \times b]\right.$ - векторное произведение в $\left.\mathbb{R}^{3}\right)$; здесь $\bar{v}_{i}, \bar{\omega}_{i}$ и $\bar{u}_{i} \in \mathbb{R}^{3} ; \beta_{i}, \bar{\rho}_{i}>0$ - некие векторные и скалярные константы, причем

$$
\bar{u}_{i} \| \bar{\omega}_{i}, \quad i=1,2 .
$$

Требуется найти такие функции $\varphi_{i} \geqslant 0$, принадлежащие пространству $C^{1}\left(\mathbb{R}^{4}\right)$, чтобы при подходящем поведении имеющихся параметров невязка $\Delta$, определенная в (7), стремилась к нулю.

Следующий раздел содержит основные результаты решения указанной задачи.

\section{2. ОСНОВНЫЕ РЕЗУЛЬТАТЫ}

Прежде чем формулировать первое утверждение, проанализируем выражения (8)-(10) с учетом условия (11), для чего несколько преобразуем их и введем удобные обозначения.

Очевидно, что в силу (11) справедливо тождество

$$
\tilde{v}_{i}^{2}=\bar{\omega}_{i}^{2} r_{i}^{2}+\left(\frac{\bar{\omega}_{i}}{\bar{\omega}_{i}^{2}}\left(\bar{\omega}_{i}, \bar{v}_{i}\right)-\bar{u}_{i} t\right)^{2}
$$

где

$$
r_{i}^{2}=\frac{1}{\bar{\omega}_{i}^{2}}\left[\bar{\omega}_{i} \times\left(x-x_{0 i}\right)\right]^{2}, \quad x_{0 i}=\frac{\left[\bar{\omega}_{i} \times \bar{v}_{i}\right]}{\bar{\omega}_{i}^{2}}, \quad i=1,2,
$$

т.е. равенство (10) приобретает вид

$$
\rho_{i}=\bar{\rho}_{i}(t, x) e^{\beta_{i} \bar{\omega}_{i}^{2} r_{i}^{2}}
$$

где введено обозначение

$$
\bar{\rho}_{i}(t, x)=\bar{\rho}_{i} \exp \left[\beta_{i}\left(\frac{\bar{\omega}_{i}}{\bar{\omega}_{i}^{2}}\left(\bar{\omega}_{i}, \bar{v}_{i}\right)-\bar{u}_{i} t\right)^{2}+2 \beta_{i} \bar{u}_{i} x\right] .
$$


Следовательно, выражение (8) можно записать так:

$$
M_{i}=\bar{\rho}_{i}(t, x) e^{\beta_{i} \bar{\omega}_{i}^{2} r_{i}^{2}}\left(\frac{\beta_{i}}{\pi}\right)^{3 / 2} e^{-\beta_{i}\left(v-\tilde{v}_{i}\right)^{2}}, \quad i=1,2 .
$$

Теперь легко видеть, что такая функция $M_{i}$ описывает вращение газа как целого с угловой скоростью $\bar{\omega}_{i}$ вокруг неподвижной оси, проходящей через точку $x_{0 i}$ (величина $r_{i}^{2}$ есть квадрат расстояния от точки $x \in \mathbb{R}^{3}$ до этой оси), а $\bar{\rho}_{i}(t, x)$ задает распределение плотности (причем $\bar{u}_{i} x$ - ее минимальное по всем $r \in[0,+\infty)$ значение при фиксированном $t$ ) вдоль оси вращения. Очевидно также, что функции (9), (15) возрастают по $t$ и $x$, причем (9) линейна по $t$, а (15) линейна по $x$ именно вдоль оси, задаваемой вектором $\bar{\omega}_{i}$ (в силу (11)), что оправдывает название "винтовые потоки", вынесенное в заголовок настоящей статьи (вектор $-\bar{u}_{i}$ играет роль “массового ускорения", а $\bar{v}_{i}$ - массовой скорости вдоль оси при $\left.t=0\right)$.

Теорема 1. Пусть распределение $f$ задается формулами (6), (8)-(11), а функици $\varphi_{i}=\varphi_{i}(t, x), i=1,2$, таковы:

$$
\varphi_{i}=\frac{D_{i}}{\left(1+t^{2}\right)^{\kappa_{i}}} C_{i}\left(x+\bar{\omega}_{i} t^{2} \frac{\bar{u}_{i}^{2}}{2 \bar{u}_{i} \bar{\omega}_{i}}\right),
$$

где $D_{i}>0, \kappa_{i} \geqslant 1 / 2$ - произвольные постоянные, $а C_{i} \geqslant 0$ - произвольные гладкие финитные или быстроубывающие функиии указанных векторных аргументов. Кроме того, предположим, что

$$
\begin{gathered}
\bar{\omega}_{i}=\bar{\omega}_{0 i} \beta_{i}^{-m_{i}}, \quad \bar{u}_{i}=\bar{u}_{0 i} \beta_{i}^{-n_{i}}, \\
\bar{v}_{i}=0,
\end{gathered}
$$

где $\bar{\omega}_{0 i}, \bar{u}_{0 i} \in \mathbb{R}^{3}$ - произвольные фиксированнъе векторь, а числа $m_{i}, n_{i}$ удовлетворяют условиям

$$
m_{i} \geqslant \frac{1}{2}, \quad n_{i} \geqslant 1
$$

Тогда определена такая величина $\Delta^{\prime}$, что $\Delta \leqslant \Delta^{\prime}$, причем существует

$$
\lim _{\beta_{1,2} \rightarrow+\infty} \Delta^{\prime}=\sum_{i=1}^{2} \bar{\rho}_{i} D_{i} K_{i}\left(\kappa_{i}\right) \sup _{x \in \mathbb{R}^{3}}\left(C_{i}(x) \mu_{i}(x)\right),
$$

где

$$
\begin{aligned}
& K_{i}\left(\kappa_{i}\right)= 2 \kappa_{i} \sup _{t \in \mathbb{R}^{1}} \frac{|t|}{\left(1+t^{2}\right)^{\kappa_{i}+1}}, \\
& \mu_{i}(x)=\left\{\begin{array}{lll}
1, & m_{i}>1 / 2, & n_{i}>1, \\
e^{2 \bar{u}_{0 i} x}, & m_{i}>1 / 2, & n_{i}=1, \\
e^{\left[\bar{\omega}_{0 i} \times x\right]^{2}}, & m_{i}=1 / 2, & n_{i}>1, \\
e^{\left[\bar{\omega}_{0 i} \times x\right]^{2}+2 \bar{u}_{0 i} x}, & m_{i}=1 / 2, & n_{i}=1 .
\end{array}\right.
\end{aligned}
$$


ДокАЗАТЕЛЬСтво. Будем использовать для максвеллианов $M_{i}, i=1,2$, входящих в (6), форму (16), учитывая, что предположение (19) приводит к существенным упрощениям в (9) и (13)-(15): мы имеем для $i=1,2$

$$
\begin{gathered}
\tilde{v}_{i}=\left[\bar{\omega}_{i} \times x\right]-\bar{u}_{i} t, \quad r_{i}^{2}=\frac{1}{\bar{\omega}_{i}^{2}}\left[\bar{\omega}_{i} \times x\right]^{2}, \quad x_{0 i}=0, \\
e^{\beta_{i} \bar{\omega}_{i}^{2} r_{i}^{2}}=e^{\beta_{i}\left[\bar{\omega}_{i} \times x\right]^{2}}, \quad \bar{\rho}_{i}(t, x)=\bar{\rho}_{i} e^{\beta_{i}\left[\bar{u}_{i}^{2} t^{2}+2 \bar{u}_{i} x\right]} .
\end{gathered}
$$

Подставим формулы (6), (16), (24), (25) в (2), (3), а затем полученные выражения в (7), учтем, что максвеллианы удовлетворяют уравнению Больцмана [1],

$$
D\left(M_{i}\right)=Q\left(M_{i}, M_{i}\right)=0, \quad i=1,2,
$$

и применим технику, развитую в работе [3]. Получим такую оценку сверху величины $\Delta$ :

$$
\begin{aligned}
\Delta \leqslant & \sup _{\substack {(t, x) \in \mathbb{R}^{4} \\
\begin{subarray}{c}{i, j=1 \\
i \neq j{ ( t , x ) \in \mathbb { R } ^ { 4 } \\
\begin{subarray} { c } { i , j = 1 \\
i \neq j } }\end{subarray}} \sum_{\mathbb{R}^{3}} d u \mid\left(\frac{\partial \varphi_{i}}{\partial t}+\left(\frac{u}{\sqrt{\beta_{i}}}+\tilde{v}_{i}\right) \frac{\partial \varphi_{i}}{\partial x}\right) e^{\beta_{i} \bar{\omega}_{i}^{2} r_{i}^{2}}+ \\
& +\varphi_{1} \varphi_{2} e^{\beta_{1} \bar{\omega}_{1}^{2} r_{1}^{2}+\beta_{2} \bar{\omega}_{2}^{2} r_{2}^{2}} \bar{\rho}_{j}(t, x) \frac{d^{2}}{\pi^{1 / 2}} \int_{\mathbb{R}^{3}} d w F_{i j}(u, t, x, w) e^{-w^{2}} \mid \bar{\rho}_{i}(t, x) \frac{e^{-u^{2}}}{\pi^{3 / 2}}+ \\
& \left.+\varphi_{1} \varphi_{2} e^{\beta_{1} \bar{\omega}_{1}^{2} r_{1}^{2}+\beta_{2} \bar{\omega}_{2}^{2} r_{2}^{2}} \bar{\rho}_{1}(t, x) \bar{\rho}_{2}(t, x) \frac{d^{2}}{\pi^{2}} \int_{\mathbb{R}^{6}} d w d u e^{-w^{2}-u^{2}} F_{i j}(u, t, x, w)\right]
\end{aligned}
$$

где

$$
F_{i j}(u, t, x, w)=\left|\frac{u}{\sqrt{\beta_{i}}}+\tilde{v}_{i}-\tilde{v}_{j}-\frac{w}{\sqrt{\beta_{j}}}\right|, \quad i \neq j .
$$

Оценку (27), очевидно, можно продолжить, опираясь на первое равенство в (24), соотношения (25) и известные свойства модулей, скалярных произведений, интегралов и точных верхних граней. В результате получаем оценку $\Delta \leqslant \Delta^{\prime}$ при

$$
\begin{aligned}
\Delta^{\prime}= & \sum_{i=1}^{2} \bar{\rho}_{i} \sup _{(t, x) \in \mathbb{R}^{4}}\left\{\left|\frac{\partial \varphi_{i}}{\partial t}\right| e^{\beta_{i}\left(\left[\bar{\omega}_{i} \times x\right]^{2}+\bar{u}_{i}^{2} t^{2}+2 \bar{u}_{i} x\right)}\right\}+ \\
& +\sum_{\substack{i, j=1 \\
i \neq j}}^{2}\left[\int_{\mathbb{R}^{3}} d u \sup _{(t, x) \in \mathbb{R}^{4}}\left\{\left(\frac{|u|}{\sqrt{\beta_{i}}}+\left|\left[\bar{\omega}_{i} \times x\right]-\bar{u}_{i} t\right|\left|\frac{\partial \varphi_{i}}{\partial x}\right|\right) \bar{\rho}_{i}(t, x) e^{\beta_{i}\left[\bar{\omega}_{i} \times x\right]^{2}}\right\} \times\right. \\
& \times \frac{e^{-u^{2}}}{\pi^{3 / 2}}+2 \frac{d^{2}}{\pi^{2}} \int_{\mathbb{R}^{6}} d w d u e^{-w^{2}-u^{2}} \sup _{(t, x) \in \mathbb{R}^{4}}\left\{\varphi_{1} \varphi_{2} e^{\beta_{1}\left[\bar{\omega}_{1} \times x\right]^{2}+\beta_{2}\left[\bar{\omega}_{2} \times x\right]^{2}} \times\right. \\
& \left.\left.\times \bar{\rho}_{1}(t, x) \bar{\rho}_{2}(t, x) F_{i j}(u, t, x, w)\right\}\right]
\end{aligned}
$$

(здесь в первой, "выделенной", сумме интегрирование по $u$ уже произведено). Прежде всего, проверим, что выражение $\Delta^{\prime}$ действительно корректно определено. Для этого, как видно из (27), достаточно доказать, что если $\varphi_{i}$ имеют вид (17), то произведения величин

$$
\varphi_{i}, \quad \frac{\partial \varphi_{i}}{\partial t}, \quad\left|\frac{\partial \varphi_{i}}{\partial x}\right|, \quad \varphi_{i}\left|\left[\bar{\omega}_{i} \times x\right]-\bar{u}_{i} t\right|, \quad\left|\frac{\partial \varphi_{i}}{\partial x}\right|\left|\left[\bar{\omega}_{i} \times x\right]-\bar{u}_{i} t\right|
$$


на множители $\rho_{i}$, заданные формулой (14) с учетом $(25)$, ограничены по $t, x$ на $\mathbb{R}^{4}$ (сходимость всех интегралов очевидна ввиду равенства (28) и присутствия в интегралах убывающих экспонент). Первое из указанных произведений после введения новой переменной

$$
y=x+\bar{\omega}_{i} t^{2} \frac{\bar{u}_{i}^{2}}{2 \bar{u}_{i} \bar{\omega}_{i}} \in \mathbb{R}^{3}
$$

приобретает вид

$$
\frac{D_{i}}{\left(1+t^{2}\right)^{\kappa_{i}}} C_{i}(y) \bar{\rho}_{i} e^{\beta_{i}\left(2 \bar{u}_{i} y+\left[\bar{\omega}_{i} \times y\right]^{2}\right)} .
$$

Следовательно, в силу свойств функций $C_{i}, i=1,2$, указанных в условии теоремы, это произведение ограничено по $y \in \mathbb{R}^{3}$, а ограниченность по $t$ вытекает из условия $\kappa_{i} \geqslant 1 / 2$. По этим же причинам ограничено четвертое произведение, после замены (31) допускающее оценку сверху величиной

$$
\frac{D_{i}}{\left(1+t^{2}\right)^{\kappa_{i}}} C_{i}(y) \bar{\rho}_{i} e^{\beta_{i}\left(2 \bar{u}_{i} y+\left[\bar{\omega}_{i} \times y\right]^{2}\right)}\left(\left|\left[\bar{\omega}_{i} \times y\right]\right|+|t|\left|\bar{u}_{i}\right|\right) .
$$

Аналогичные соображения показывают ограниченность и остальных трех произведений, поскольку

$$
\begin{aligned}
\frac{\partial \varphi_{i}}{\partial x}= & \frac{D_{i}}{\left(1+t^{2}\right)^{\kappa_{i}}} C_{i}^{\prime}\left(x+\bar{\omega}_{i} t^{2} \frac{\bar{u}_{i}^{2}}{2 \bar{u}_{i} \bar{\omega}_{i}}\right) \\
\frac{\partial \varphi_{i}}{\partial t}= & -\frac{2 t \kappa_{i} D_{i}}{\left(1+t^{2}\right)^{\kappa_{i}+1}} C_{i}\left(x+\bar{\omega}_{i} t^{2} \frac{\bar{u}_{i}^{2}}{2 \bar{u}_{i} \bar{\omega}_{i}}\right)+ \\
& +\frac{D_{i} t}{\left(1+t^{2}\right)^{\kappa_{i}}}\left(C_{i}^{\prime}\left(x+\bar{\omega}_{i} t^{2} \frac{\bar{u}_{i}^{2}}{2 \bar{u}_{i} \bar{\omega}_{i}}\right), \bar{\omega}_{i}\right) \frac{\bar{u}_{i}^{2}}{\bar{u}_{i} \bar{\omega}_{i}} .
\end{aligned}
$$

Найдем теперь предел суммы (29) при $\beta_{1,2} \rightarrow+\infty$. Для этого еще раз перепишем eе, используя (18), (19), выражение для $\tilde{v}_{i}$ в $(24)$, формулы $(28),(31),(34)$ и вводя новые обозначения $\gamma_{i}=1 / \beta_{i}, i=1,2$. После этой замены надо искать предел при $\gamma_{1,2} \rightarrow 0$ выражения

$$
\begin{aligned}
\Delta^{\prime}= & \sum_{i=1}^{2} \bar{\rho}_{i} D_{i} \sup _{(t, y) \in \mathbb{R}^{4}}\left\{\frac{|t|}{\left(1+t^{2}\right)^{\kappa_{i}}} C_{i}(y) e^{2 \bar{u}_{0 i} y \gamma_{i}^{n_{i}-1}+\left[\bar{\omega}_{0 i} \times y\right]^{2} \gamma_{i}^{2 m_{i}-1}} \times\right. \\
& \left.\times\left|\frac{2 \kappa_{i}}{1+t^{2}}+\left(C_{i}^{\prime}(y), \bar{\omega}_{0 i}\right) \frac{\bar{u}_{0 i}^{2}}{\bar{u}_{0 i} \bar{\omega}_{0 i}} \gamma_{i}^{n_{i}}\right|\right\}+ \\
& +\sum_{\substack{i, j=1 \\
i \neq j}}^{2}\left[\int _ { \mathbb { R } ^ { 3 } } d u \operatorname { s u p } _ { ( t , y ) \in \mathbb { R } ^ { 4 } } \left\{\left(|u| \sqrt{\gamma_{i}}+\left|\gamma_{i}^{m_{i}}\left[\bar{\omega}_{0 i} \times y\right]-\gamma_{i}^{n_{i}} \bar{u}_{0 i} t\right| \times\right.\right.\right. \\
& \left.\left.\times \frac{D_{i}}{\left(1+t^{2}\right)^{\kappa_{i}}} C_{i}^{\prime}(y)\right) \bar{\rho}_{i} e^{2 \bar{u}_{0 i} y \gamma_{i}^{n_{i}-1}+\left[\bar{\omega}_{0 i} \times y\right]^{2} \gamma_{i}^{2 m_{i}-1}}\right\} \frac{e^{-u^{2}}}{\pi^{3 / 2}}+ \\
& +2 \frac{d^{2}}{\pi^{2}} \int_{\mathbb{R}^{6}} d w d u e^{-w^{2}-u^{2}} \sup _{(t, y) \in \mathbb{R}^{4}}\left\{\frac{D_{1} D_{2}}{\left(1+t^{2}\right)^{\kappa_{1}+\kappa_{2}}} C_{1}(y) C_{2}(y) \bar{\rho}_{1} \bar{\rho}_{2} \times\right.
\end{aligned}
$$




$$
\begin{aligned}
& \times \exp \left(2 y\left(\bar{u}_{01} \gamma_{1}^{n_{1}-1}+\bar{u}_{02} \gamma_{2}^{n_{2}-1}\right)+\sum_{i=1}^{2}\left[\bar{\omega}_{0 i} \times y\right]^{2} \gamma_{i}^{2 m_{i}-1}\right) \times \\
& \left.\left.\times\left|u \sqrt{\gamma_{i}}+\left[\bar{\omega}_{0 i} \times y\right] \gamma_{i}^{m_{i}}-\bar{u}_{0 i} t \gamma_{i}^{n_{i}}-\left[\bar{\omega}_{0 j} \times y\right] \gamma_{j}^{m_{j}}+\bar{u}_{0 j} t \gamma_{j}^{n_{j}}-w \sqrt{\gamma_{j}}\right|\right\}\right] .
\end{aligned}
$$

Легко видеть, что в данной сумме все слагаемые, кроме первого, представляют собой произведения тех или иных положительных (в силу неравенств (20)) степеней величин $\gamma_{i}, i=1,2$, на конечные величины (существование конечных точных верхних граней и интегралов обосновано выше), и поэтому стремятся к нулю. Что касается оставшегося первого слагаемого, то входящая в него точная верхняя грань по $t$ и $y$, очевидно, распадается на произведение отдельных точных верхних граней по каждой из переменных, что с использованием обозначения (22) дает

$$
\sum_{i=1}^{2} \bar{\rho}_{i} D_{i} K\left(\kappa_{i}\right) \sup _{y \in \mathbb{R}^{3}}\left\{C_{i}(y) e^{2 \bar{u}_{0 i} y \gamma_{i}^{n_{i}-1}+\left[\bar{\omega}_{0 i} \times y\right]^{2} \gamma_{i}^{2 m_{i}-1}}\right\} .
$$

K последней точной верхней грани может быть применена лемма 1 из [3], условия которой легко проверяются на основании условий нашей теоремы. Это обеспечивает непрерывность точной верхней грани как функции от $\gamma_{1,2}$ в достаточно малой окрестности нуля. Таким образом, в (36) можно перейти к пределу при $\gamma_{1,2} \rightarrow 0$ под знаком sup, в результате чего получим четыре возможных предельных значения в зависимости от величин $m_{i}, n_{i}, i=1,2$, ограниченных условиями (20). Итак, мы с точностью до обозначений приходим к соотношениям (21)-(23), что и требовалось. Теорема доказана.

СлеДСТВИЕ 1. При выполнении условий теоремы 1 справедливо следующее утверждение: для произвольного положительного в найдется $\delta>0$ такое, что при всех $D_{1}, D_{2}$, меньших $\delta$, д достаточно больших значениях $\beta_{1}, \beta_{2}$ выполнено неравенство $\Delta<\varepsilon$.

ДокАЗАТЕЛЬСТво очевидно в силу соотношения (21), ибо входящие в него константы $\bar{\rho}_{i}, K_{i}\left(\kappa_{i}\right)$ и точные верхние грани конечны (благодаря свойствам функций $\left.C_{i}, i=1,2\right)$ и не зависят от $D_{1}, D_{2}$, а предельный переход по $\beta_{1,2} \rightarrow+\infty$ уже осуществлен.

Следующий результат содержит дополнительное предположение о структуре коэффициентных функций $\varphi_{i}, i=1,2$, сходное с использованным ранее в [3], [11], [14].

Теорема 2. Пусть коэфбициенты $\varphi_{i}, i=1,2$, в (6) имеют вид

$$
\varphi_{i}(t, x)=\psi_{i}(t, x) \bar{\rho}_{i}\left[\bar{\rho}_{i}(t, x)\right]^{-1} e^{-\beta_{i} \bar{\omega}_{i}^{2} r_{i}^{2}},
$$

где $r_{i}^{2}$ и $\bar{\rho}_{i}(t, x)$ заданъь в (13), (15). Пусть при

$$
m_{i}>\frac{1}{2}, \quad n_{i}>1
$$

вновъ выполнены предположения (18). Тогда, если множители $\psi_{i}, i=1,2$, имеют вид

$$
\psi_{i}(t, x)=D_{i} C_{i}(t) E_{i}(x)
$$


где $C_{i}, E_{i} \geqslant 0$ - некие гладкие финитные или быстроубъвающие функиии, то справедливо неравенство $\Delta<\varepsilon$.

ДоказАтельство. Снова используем представление (16), однако ввиду отсутствия теперь предположения (19) следует вычислять входящие в (16) величины по исходным формулам (9), (13), (15), а не по (24), (25). Поэтому выкладки, аналогичные проделанным выше при доказательстве теоремы 1, вновь приводят (в силу (11), (26) и т.п.) к соотношениям (27), (28), однако с другими значениями для $\bar{\rho}_{i}(t, x), \tilde{v}_{i}, r_{i}^{2}, i=1,2$. Далее, подставим в (27) выражения (36) вместо (17) и учтем формулы (15) и тот факт, что теперь производные (34) выглядят совершенно иначе:

$$
\begin{aligned}
\frac{\partial \varphi_{i}}{\partial x} & =\left(\frac{\partial \psi_{i}}{\partial x}-2 \beta_{i} \psi_{i}\left(\bar{u}_{i}+\left[\left[\bar{\omega}_{i} \times\left(x-x_{0 i}\right)\right] \times \bar{\omega}_{i}\right]\right)\right) \varphi_{i} \psi_{i}^{-1} \\
\frac{\partial \varphi_{i}}{\partial t} & =\left(\frac{\partial \psi_{i}}{\partial t}+2 \beta_{i} \psi_{i}\left(\frac{\bar{\omega}_{i}}{\bar{\omega}_{i}^{2}}\left(\bar{\omega}_{i}, \bar{v}_{i}\right)-\bar{u}_{i} t\right) \bar{u}_{i}\right) \varphi_{i} \psi_{i}^{-1} .
\end{aligned}
$$

В результате получим следующее выражение для величины $\Delta^{\prime}$ в оценке $\Delta \leqslant \Delta^{\prime}$ :

$$
\begin{aligned}
\Delta^{\prime}= & \sum_{\substack{i, j=1, i \neq j}}^{2}\left[\bar{\rho}_{i} \frac{1}{\pi^{3 / 2}} \int_{\mathbb{R}^{3}} d u e^{-u^{2}} \sup _{(t, x) \in \mathbb{R}^{4}} \mid \frac{\partial \psi_{i}}{\partial t}+2 \beta_{i} \psi_{i}\left(\frac{\left(\bar{\omega}_{i}, \bar{u}_{i}\right)}{\bar{\omega}_{i}^{2}}\left(\bar{\omega}_{i}, \bar{v}_{i}\right)-t \bar{u}_{i}^{2}\right)+\right. \\
& +\left(\frac{\partial \psi_{i}}{\partial x}-2 \beta_{i} \psi_{i}\left(\bar{u}_{i}+\left[\left[\bar{\omega}_{i} \times\left(x-x_{0 i}\right)\right] \times \bar{\omega}_{i}\right]\right)\right)\left(\frac{u}{\sqrt{\beta_{i}}}+\tilde{v}_{i}\right)+ \\
& +\psi_{1} \psi_{2} \bar{\rho}_{j} \frac{d^{2}}{\pi^{1 / 2}} \int_{\mathbb{R}^{3}} d w F_{i j} e^{-w^{2}} \mid e^{-u^{2}} d u+ \\
& \left.+\bar{\rho}_{1} \bar{\rho}_{2} \frac{d^{2}}{\pi^{2}} \int_{\mathbb{R}^{3}} d w d u e^{-w^{2}-u^{2}} \sup _{(t, x) \in \mathbb{R}^{4}}\left(\psi_{1} \psi_{2} F_{i j}\right)\right] .
\end{aligned}
$$

Здесь $F_{i j}$ также задаются формулой $(28)$, однако $\tilde{v}_{i}$ имеют вид $(9)$. Конечность величины (41) обеспечивается тем, что если выполнено (39), то все функции (30) (с заменой $\varphi_{i}$ на $\left.\psi_{i}, i=1,2\right)$, очевидно, ограничены на $\mathbb{R}^{4}$. Предельный переход в (41) по $\beta_{1,2} \rightarrow+\infty$ с использованием (18) при условии (38) дает (возможность предельного перехода обосновывается точно так же, как при доказательстве теоремы 1)

$$
\begin{aligned}
\lim _{\beta_{1,2} \rightarrow+\infty} \Delta^{\prime}= & \sum_{\substack{i, j=1, i \neq j}}^{2} \bar{\rho}_{i} \sup _{(t, x) \in \mathbb{R}^{4}}\left|\frac{\partial \psi_{i}}{\partial t}+\bar{v}_{i} \frac{\partial \psi_{i}}{\partial x}+\bar{\rho}_{j} \pi d^{2} \psi_{1} \psi_{2}\right| \bar{v}_{i}-\bar{v}_{j}||+ \\
& +2 \pi d^{2} \bar{\rho}_{1} \bar{\rho}_{2}\left|\bar{v}_{1}-\bar{v}_{2}\right| \sup _{(t, x) \in \mathbb{R}^{4}}\left(\psi_{1} \psi_{2}\right),
\end{aligned}
$$

ибо теперь $F_{i j}, i \neq j$, вместо нулевого предела имеют

$$
\lim _{\beta_{1,2} \rightarrow+\infty} F_{i j}=\left|\bar{v}_{1}-\bar{v}_{2}\right|
$$


Наконец, подставим (39) в (41), получим

$$
\begin{aligned}
\lim _{\beta_{1,2} \rightarrow+\infty} \Delta^{\prime}= & \sum_{\substack{i, j=1, i \neq j}}^{2} D_{i} \bar{\rho}_{i} \sup _{(t, x) \in \mathbb{R}^{4}} \mid C_{i}^{\prime}(t) E_{i}(x)+C_{i}(t) \bar{v}_{i} E_{i}^{\prime}(x)+ \\
& +D_{j} \bar{\rho}_{j} \pi d^{2} C_{i}(t) C_{j}(t) E_{i}(x) E_{j}(x)\left|\bar{v}_{i}-\bar{v}_{j}\right| \mid+ \\
& +2 \pi d^{2} D_{1} D_{2} \bar{\rho}_{1} \bar{\rho}_{2}\left|\bar{v}_{1}-\bar{v}_{2}\right| \sup _{t \in \mathbb{R}^{1}}\left(C_{1} C_{2}\right) \sup _{x \in \mathbb{R}^{3}}\left(E_{1} E_{2}\right),
\end{aligned}
$$

откуда с учетом $\Delta \leqslant \Delta^{\prime}$ немедленно следует $\Delta<\varepsilon$. Теорема доказана.

ЗАмЕчАниЕ 1 . В теореме 2 скорости $\bar{v}_{i}$ произвольны, в отличие от теоремы 1 , где предполагалось (19), зато на коэффициентные функции $\varphi_{i}$ и векторы $\bar{\omega}_{i}, \bar{u}_{i}$ налагаются более жесткие ограничения (37)-(39), чем (17), (20).

ЗАмЕчАниЕ 2. Представляется возможным увеличить количество ситуаций, рассматривая в теореме 2, помимо случая (38), и другие сочетания условий на $m_{i}, n_{i}$, $i=1,2$, возникающие в (20), подобно тому, как это сделано в (23). Более того, условия (20) можно было бы ослабить (см., например, [3]). Это привело бы к некоторому усложнению правой части в (42) и самого доказательства, но не дало ничего принципиально нового по сравнению с окончательным неравенством $\Delta<\varepsilon$.

Таким образом, в настоящей работе нам удалось построить в явном виде некоторые новые приближенные решения уравнения Больцмана в случае модели твердых сфер. Они найдены в виде бимодальных распределений, т.е. линейных комбинаций локально максвелловских решений, соответствующих винтовым потокам с ускорением и уплотнением, имеющих вид (8)-(11). Выбор коэффициентных функций этих распределений в специальной форме (17) либо (37), (39) позволяет при некоторых дополнительных условиях на поведение угловых и линейных скоростей потоков, а также их температур и других параметров сделать “смешанную" невязку между частями этого уравнения сколь угодно малой.

\section{Список литературы}

[1] К. Черчиньяни, Теория и приложения уравнения Больимана, Мир, М., 1978.

[2] Т. Карлеман, Математические задачи кинетической теории газов, ИЛ, М., 1960.

[3] В. Д. Гордевский, ТМФ, 126:2 (2001), 283-300.

[4] H. Grad, Comm. Pure and Appl. Math., 2:4 (1949), 331-407.

[5] О. Г. Фридлендер, Прикл. матем. и мех., 29:5 (1965), 973.

[6] М.Н. Коган, Динамика разреженного газа. Кинетическая теория, Наука, М., 1967.

[7] V. D. Gordevskyy, Math. Methods Appl. Sci., 27:2 (2004), 231-247.

[8] В. Д. Гордевский, Матем. физ., анализ, геом., 2:2 (1995), 168-176.

[9] В. Д. Гордевский, Матем. физ., анализ, геом., 4:1-2 (1997), 46-58.

[10] В. Д. Гордевский, ТМФ, 114:1 (1998), 126-136.

[11] V.D. Gordevskyy, Nonlinear Anal., 53:3-4 (2003), 481-494.

[12] V.D. Gordevsky, Math. Methods Appl. Sci., 21:16 (1998), 1479-1494.

[13] В. Д. Гордевский, ТМФ, 135:2 (2003), 303-314.

[14] В. Д. Гордевский, Н. В. Андрияшева, Матем. физ., анализ, геом., 5:1 (2009), 38-53. 